\title{
Joint beamforming and power allocation for wireless powered UAV-assisted cooperative NOMA systems
}

\author{
Guangchen Mu
}

\begin{abstract}
In this paper, we investigate an unmanned aerial vehicle (UAV)-enabled non-orthogonal multiple access (NOMA) systems, where UAV acts as a full-duplex (FD) relay to help the communication between the base station (BS) and two NOMA users. Assume that the UAV follows a circular trajectory and applies decode-and-forward (DF) strategy. Using simultaneous wireless information and power transfer (SWIPT), the UAV harvests energy from the BS in the first time slot and self-interference due to FD mode in the second time slot. By the joint optimization of beamforming and time allocation ratio, we aim at maximizing sum throughput of the whole system and harvested energy at UAV. To solve two highly non-convex problem, we propose the corresponding algorithms based on inner approximation method, respectively, which can converge to at least optimal solutions in few steps. In terms of two different system performances, numerical results can verify that the effectiveness of the proposed scheme. We also find the optimal azimuth angle of UAV's circular trajectory by simulation.
\end{abstract}

Keywords: Unmanned aerial vehicle (UAV), Non-orthogonal multiple access (NOMA), Simultaneous wireless information and power transfer (SWIPT), Full-duplex relay

\section{Introduction}

With high mobility deployment flexibility and costeffectiveness, the application of unmanned aerial vehicles (UAVs) in wireless communication has drawn wide attention in academia, research industries, and government [1-3]. UAV-enabled wireless network is recognized as a potential technique to provide communication service in natural disasters such as flood or earthquake [4]. To further enhance coverage and connectivity in some particular cases, many research efforts about UAV-enabled communication system have been made from the perspective of UAVs' different roles, i.e., as a fixed base source and a demand relay [5].

As substitution of static relay, UAV can adjust its position dynamically according to the current environment so that performance gain of system is improved [6-8]. In [6], the author considered amplify-and-forward (AF) UAVassisted relaying system in terms of outage probability.

Correspondence: hngxymgc@126.com

Henan Institute of Technology, School of Electronic Information Engineering, PingYuan Road 669, 453003 XinXiang, China
In [7], UAV is regarded as the relay to help communication between nodes in device-to-device network. The [8] proposed scheme maximized the end-to-end throughput by jointly optimizing power and UAV trajectory in UAVassisted relaying system. However, the aforementioned literatures focus on half-duplex (HD) relaying, which leads to the loss of spectral efficiency due to extra cooperative transmission time [9].

As two potential techniques in next generation communication systems, full-duplex relaying [10, 11]and non-orthogonal multiple access (NOMA) [12, 13] are devoted to improving spectral efficiency. On the one hand, FD relaying allows a relay node to transmit and receives simultaneously at the same band different from HD mode. However, severe self-interference (SI) brings great challenge to the feasibility of FD relaying systems. To overcome this problem, the researchers have proposed some effective approaches, such as antenna isolation and analog/digital cancelation $[14,15]$. As for FD relaying, there are two types of transmission protocols, namely decode-and-forwarding (DF) and amplify-andforwarding (AF) $[16,17]$. Recently, many literatures are 
proposed to further improve spectral efficiency [19-21]. $\mathrm{Yu}$ et al. [18] further studied outage probability minimization by optimizing the location of relay in full-duplex relaying system. Afterwards, the author considered the joint optimization of transmit power at source/relay and the location of relay in terms of outage probability [20]. Moreover, [21] demonstrated that the full-duplex monitor can significantly improve eavesdropping rate. However, in [19-21], the position of the relay is fixed, which is not suitable for the UAV mobile relay. Inspired by this, [22] considered a more general case where the static relay is replaced by UAV for cooperative communication. A novel UAV-enabled full-duplex scheme for the minimizing sum outage probability in relaying system is proposed. In [23], a UAV-enabled full-duplex relaying system was studied. The authors verified the superiority of the proposed scheme in terms of instantaneous data rate and outage probability.

On the other hand, non-orthogonal multiple access (NOMA) can not only significantly improve the spectrum efficiency but also allow more users or devices to access the network [24-29]. Different from the conventional orthogonal multiple access (OMA), the core of NOMA is to utilize superposed coding (SC) at the transmitter and successive interference cancelation (SIC) at the receivers [24]. The NOMA users are served on the same resource block, such as time/frequency/code [26, 27]. The superior performance of NOMA has stimulated many scholars' interests, especially cooperative NOMA schemes [28, 29]. In [28], a novel full-duplex cooperative NOMA system was investigated, where the user in good channel condition was regarded as the relay to help communication between the source and the user in poor channel condition. Additionally, the authors maximized the data rate of relay user under quality-of-service (QoS) requirement of the user in poor channel condition. Liu et al. [29] induced a dedicated relay to assist the BS for data transmission. The paper is dedicated to maximizing energy efficiency of system by the joint optimization of transmit power at the source/relay. Wu et al. [28] and [29] both showed the superiority of the proposed cooperative schemes by simulation.

Motivated by the advantage of NOMA, more and more scholars are trying to apply the NOMA to UAV-enabled network for better performance [30]. Sun et al. [31] proposed a novel iterative algorithm to maximize minimum throughput by jointly optimizing multiuser communication scheduling with cyclical NOMA and UAV trajectory. To improve user fairness, [32] integrated NOMA and UAV into the satellite network. The authors proposed a power allocation scheme to improve outage performance. Qi et al. [33] considered sum rate maximization problem as a function of power allocation and UAV altitude. Simulation results verified that the UAV-enabled network with
NOMA scheme can bring more performance gain over the existing schemes.

UAV-enabled wireless networks are facing some challenges while bringing significant performance gain. One of critical issues is the energy-constrained battery of UAV which would not support wireless communication constantly. Moreover, the lifetime of network would not be prolonged insufficiently by adjusting the UAV's locations and the power allocation. Instead of decreasing energy consumption by maximizing energy efficiency [34, 35], simultaneous wireless information and power transfer (SWIPT) is recognized as a more effective approach to alleviate energy consumption problem [36-38]. The core of SWIPT is to enable the wireless devices to harvest energy from the radio frequency signal while receiving the signal. The existing receiver architecture of SWIPT is divided into two categories, i.e., time switching (TS) and power splitting (PS) $[39,40]$. Motivated by this, some literatures about the application of SWIPT to UAV-enabled wireless network are studied [41-43]. Yin et al. [41] investigated the end-to-end throughput maximization for UAV-assisted cooperative communication system, where the UAV act as a dedicated relay to help data transmission between the BS and the destination by employing PS protocol. To solve this problem, the author alternately solved the two subproblems by the joint optimization of the UAV's power profile, power-splitting ratio profile, and trajectory. In [42], the wireless powered UAVassisted cooperative communication system was studied. The author verified the effectiveness of proposed scheme in terms of outage probability and throughput of system. $\mathrm{Xu}$ et al. [43] studied UAV-assisted wireless communication system where the UAV is charged by two energy receivers on the ground. By jointly optimizing the altitude, trajectory, and transmit beam-width of the UAV, the energy harvested from two energy receivers was maximized.

Different from the above literatures, we consider the application of NOMA and full-duplex relaying to UAVassisted wireless network. On the one hand, the BS employs the NOMA protocol for data transmission and UAV operates in FD mode to implement the relaying behavior so that spectral efficiency is enhanced. On the other hand, we pay attention to the time switching mechanism to enable the UAV to harvest energy and relay data in two phases respectively such that communication reliability is improved.

The contributions of this paper are summarized as follows:

- A UAV-enabled cooperative NOMA system with application of SWIPT is studied in this paper, where the wireless powered UAV files along the fixed circle at the fixed height. Under power constraints at the BS and UAV as well as QoS requirement of two NOMA users, 
we formulate two optimization problems such that sum throughput of system and the harvested energy at UAV are maximized.

- Given the circular trajectory of UAV's fight, we propose an iterative algorithm to tackle such problem by the joint optimization of transmit beamforming vectors and time allocation coefficient. With low complexity and fast convergence, the algorithm guarantees to reach at least local optimum within few iterations.

- We demonstrate that the proposed scheme is superior to other baseline schemes by simulation results in terms of sum throughput of system. We also consider the impact of location of UAV on the harvested energy at UAV. In addition, the optimal azimuth angle of UAV's fight can be obtained by simulation.

Organization: The rest of the paper is organized as follows. Section 2 describes the UAV-enabled cooperative NOMA system with the application of SWIPT in detail and formulates the optimization problem mathematically. In Section 3, a low-complexity iterative algorithm is proposed to solve the highly non-convex problem by the joint optimization of beamforming vectors and time allocation ratio. Simulation results and conclusion are presented in Sections 4 and 5 , respectively.

Notations: In this paper, the bold capital and lower-case letters are used to denote matrices and vectors, respectively. $R\{\bullet\}$ stands for the real part of a variable. $\varepsilon\{\cdot\}$, $(\cdot)^{H}, \operatorname{Tr}(\cdot)$, and rank $(\cdot)$ indicate the expectation, Hermitian transpose, trace, and rank, respectively. $\mathbf{I}_{N}$ denotes the $N \times N$ identity matrix; $\mathbb{C}^{N \times M}$ and $\mathbb{H}_{+}^{N}$ denote the $N \times M$ complex matrices and $N \times N$ Hermitian matrices, respectively. $\|\cdot\|_{2}$ means the Euclidean norm of a vector while $\|\cdot\|_{F}$ means the Frobenius norm of a matrix; null (.) denotes the null space of a vector or matrix. $\mathbf{x} \sim$ $\mathcal{C N}(\mu, \Lambda)$ means the vector $\mathbf{x}$ is a complex Gaussian variable with mean $\mu$ and covariance $\Lambda$.

\section{System model and problem formulation}

\subsection{System model}

Figure 1 illustrates a wireless powered UAV-enabled relaying communication system with a full-duplex (FD) UAV, a base station (BS), and two downlink users. Using NOMA, the BS is intended to serve two users with different levels of power. Assume that the BS is unable to deliver the superimposed signals to two NOMA users owing to severe blockage. As shown in Fig. 1, high mobility UAV acts as a decode and forward (DF) relay to help data transmission between the BS and two users. In addition, the UAV can harvest energy from the BS and self-interference with utilization of time-splitting (TS) protocol to support relaying behavior. Assume that the $\mathrm{BS}$ is equipped with $M>1$ transmit antennas and two NOMA users are both single-antenna devices. The UAV operates in FD mode with a set of one receive antenna and $N>1$ transmit antenna. Assume that all channel state information is perfectly known [44].

As shown in Fig. 2, the whole communication block $T$ is divided into two slots. In the first time slot $\alpha T$, the UAV harvests the energy from the BS. In the second time slot $(1-\alpha) T$, the UAV receives the signal from the BS and transmits the superposed signal to two NOMA users. For simplicity, $T$ is set as 1 . In addition, the UAV would harvest energy from self-interference during the second period. The harvested energy at UAV is used for relaying behavior.

\subsection{Channel model}

Without loss of generality, we consider a threedimensional (3D) Cartesian coordinate system model. As illustrated in Fig. 1, the BS, NOMA near user, and far NOMA user are located at $\left(-d_{s}, 0,0\right),\left(-d_{1}, 0,0\right)$, and $\left(-d_{2}, 0,0\right)$ respectively, where $d_{2}>d_{1}=d_{s}=d>0$. UAV is deployed at fixed height $h$ and flies in circular domain with radius of $r$, whose center is located at $(0,0, h)$. In addition, the corresponding azimuth angle while UAV is flying along the circular trajectory is denoted by $0 \leq \theta \leq 2 \pi$. Hence, the fight trajectory of UAV can be written as $(r \cos \theta, r \sin \theta, h)$. In practice, the channels between UAV and two users as well as the BS the channel contain two components, i.e., line-of-sight (LoS) and the non-LoS (NLoS), where LoS channel is dominant [45]. Thus, all channels can be modeled as

$$
\mathbf{h}_{i}=\beta \tilde{\mathbf{h}}_{i} / d_{i}^{\tau_{i}(\theta)}(\theta) i \in\{S R, R 1, R 2\},
$$

where $\beta$ stands for the channel power gain at the reference distance $1 \mathrm{~m} ; \mathbf{h}_{S R}, \mathbf{h}_{R 1}$, and $\mathbf{h}_{R 2}$ denote channel BS-UAV, channel UAV-U1, and UAV-U2, respectively. Note that $\mathbf{h}_{S R}, \mathbf{h}_{R 1}$, and $\mathbf{h}_{R 2}$ are denoted by

$\tilde{\mathbf{h}}_{i}=\sqrt{\frac{K_{i}(\theta)}{K_{i}(\theta)+1}} \tilde{\mathbf{h}}_{i}^{L o S}+\sqrt{\frac{1}{K_{i}(\theta)+1}} \tilde{\mathbf{h}}_{i}^{N L o S}, i \in\{S R, R 1, R 2\}$

where $\tilde{\mathbf{h}}_{i}^{L o S}$ represents LoS component which satisfies $\operatorname{Tr}\left\{\tilde{\mathbf{h}}_{S R}^{L O S}\left(\tilde{\mathbf{h}}_{S R}^{L O S}\right)^{H}\right\}=M, \quad \operatorname{Tr}\left\{\tilde{\mathbf{h}}_{R 1}^{L O S}\left(\tilde{\mathbf{h}}_{R 1}^{L O S}\right)^{H}\right\}=N$, $\operatorname{Tr}\left\{\tilde{\mathbf{h}}_{R 2}^{L o S}\left(\tilde{\mathbf{h}}_{R 2}^{L o S}\right)^{H}\right\}=N$. And a Rayleigh fading channel component, which $\tilde{\mathbf{h}}_{i}^{N L O S}$ is assumed to be independently circularly symmetric complex Gaussian (CSCG), is distributed with zero mean and unit variance, i.e., $\tilde{\mathbf{h}}_{i}^{N L o S} \sim \mathcal{C N}(\mathbf{0}, \mathbf{I})$. The Rician factor $K_{i}(\theta)$ can be given by $K_{i}(\theta)=v_{1} e^{\mu_{1} \varpi_{i}(\theta)}$, where $\nu_{1}$ and $\mu_{1}$ are constant coefficients and $\varpi_{i}(\theta)$ is fight angle of UAV relative to communication devices, i.e., $\varpi_{i}(\theta)=\arcsin \left(h / d_{i}\right), i \in\{S R, R 1, R 2\} . d_{i}, i \in\{S R, R 1, R 2\}$ 


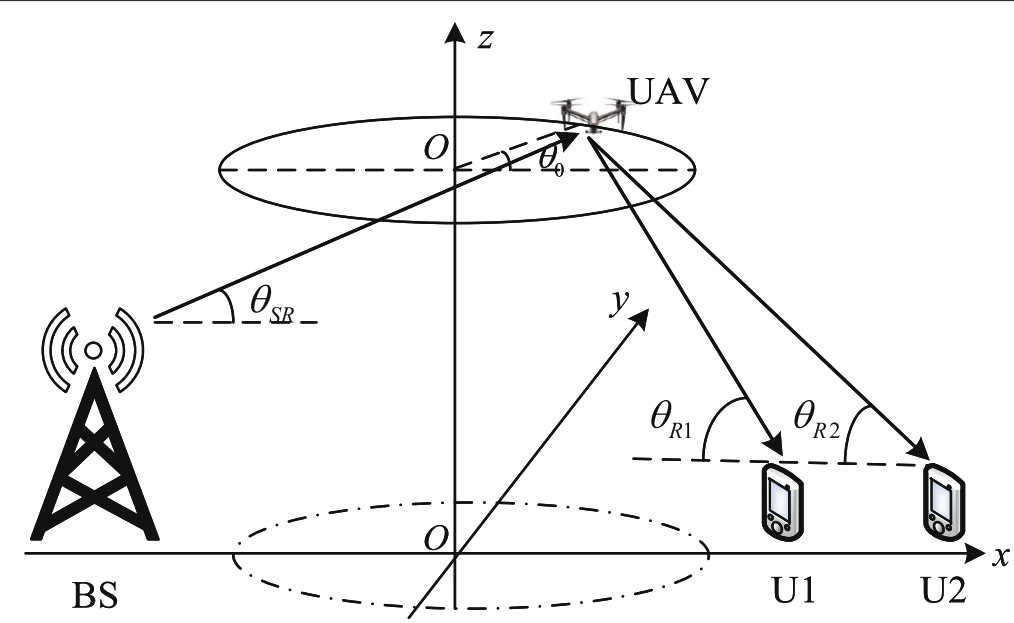

Fig. 1 Full-duplex wireless powered UAV-aided cooperative NOMA network

represents the distances between UAV and all nodes, which are given by

$$
\begin{aligned}
& d_{S R}=\sqrt{h^{2}+\left(d_{S R}+r \cos \theta\right)^{2}}, \\
& d_{R 1}=\sqrt{h^{2}+\left(d_{R 1}-r \cos \theta\right)^{2}} \\
& d_{R 2}=\sqrt{h^{2}+\left(d_{R 2}-r \cos \theta\right)^{2}}
\end{aligned}
$$

In addition, $\tau_{i}(\theta)$ is path loss exponent which is written as $\tau_{i}(\theta)=v_{2} P_{L o S}\left(\varpi_{i}(\theta)\right)+\nu_{3}$ with LoS probability $P_{L o S}\left(\varpi_{i}(\theta)\right)=\left(1+v_{4} e^{-u_{2} \varpi_{i}(\theta)}\right)^{-1}$. Herein, $v_{2}, v_{3}, v_{4}$, and $\mu_{2}$ are environment constants.

\subsection{Signal model}

According to NOMA principle, the BS transmits the superimposed signal $\mathbf{x}(n)=\mathbf{w}_{1} x_{1}(n)+\mathbf{w}_{2} x_{2}(n)$ to UAV, where $\mathbb{E}\left\{\left|x_{1}\right|^{2}\right\}=\mathbb{E}\left\{\left|x_{2}\right|^{2}\right\}=1$ and $\left\|\mathbf{w}_{1}\right\|^{2}+\left\|\mathbf{w}_{2}\right\|^{2} \leq$ $P_{s}$. Then, the UAV forwards the decoded mixed signal $\mathbf{s}(n)=\mathbf{s}_{1} \hat{x}_{1}(n+\kappa)+\mathbf{s}_{2} \hat{x}_{2}(n+\kappa)$ to two users using NOMA, where $\kappa$ is time delay; $\hat{x}_{1}(n+\kappa), \hat{x}_{2}(n+\kappa)$ are the decoded versions of $x_{1}(n), x_{2}(n)$, and $\left\|\mathbf{s}_{1}\right\|^{2}+\left\|\mathbf{s}_{2}\right\|^{2}$ is no more than the maximum transmission power at UAV.

In the first phase $\alpha T$, the harvested energy at UAV from the BS can be expressed as

$$
E_{1}=\eta \phi_{1}(\theta)\left(\left|\mathbf{h}_{S R}^{H} \mathbf{w}_{2}\right|^{2}+\left|\mathbf{h}_{S R}^{H} \mathbf{w}_{1}\right|^{2}\right) \alpha T
$$

where $\eta \in[0,1]$ is the energy conversion efficiency at UAV and $\phi_{1}(\theta)=\frac{\beta}{d_{S R}^{\tau}{ }^{(\theta)}(\theta)}$.

In the second time phase $(1-\alpha) T$, the received signal from the BS at the UAV is expressed as

$$
\mathrm{y}_{r}=\phi_{2}(\theta) \mathbf{h}_{S R}^{H} \mathbf{x}(n)+\sqrt{\rho} \mathbf{h}_{r r}^{H} \mathbf{s}(n)+n_{r}
$$

where $\phi_{2}(\theta)=\sqrt{\frac{\beta}{d_{S R}^{\tau S R}(\theta)}}, \rho$ represents the SI cancelation level and $n_{r} \sim \mathcal{C N}\left(0, \sigma_{r}^{2}\right)$ is the noise at UAV.

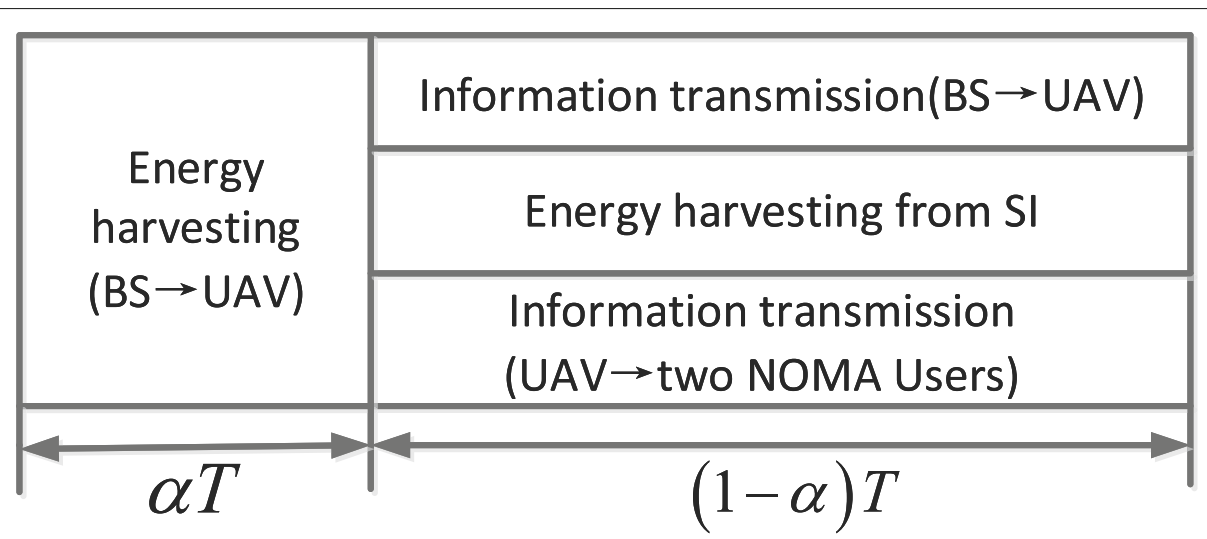

Fig. 2 An illustration of time block diagram of the energy harvesting and information transmission in TS protocol 
Meanwhile, the UAV forwards the decoded signals to two NOMA users. Then, the received signal at U1 and U2 can be denoted by

$$
y_{1}=\phi_{3}(\theta) \mathbf{h}_{R 1}^{H} \mathbf{s}(n)+n_{1}
$$

and

$$
y_{2}=\phi_{4}(\theta) \mathbf{h}_{R 2}^{H} \mathbf{s}(n)+n_{2}
$$

respectively, where $\sqrt{\frac{\beta}{d_{R 1}^{\tau_{R 1}(\theta)}(\theta)}}, \sqrt{\frac{\beta}{d_{R 2}^{\tau_{R 2}(\theta)}(\theta)}}, \quad n_{1}$ $\mathcal{C N}\left(0, \sigma_{1}^{2}\right)$, and $n_{2} \sim \mathcal{C N}\left(0, \sigma_{2}^{2}\right)$ are noise at U1 and U2 respectively.

According to [11], the portion of SI filtered by the receiver filter proposed can be utilized for energy harvesting; we have

$$
E_{S I}=\eta\left(\left|\mathbf{h}_{r r}^{H} \mathbf{s}_{2}\right|^{2}+\left|\mathbf{h}_{r r}^{H} \mathbf{s}_{1}\right|^{2}\right)(1-\alpha) T
$$

Thus, the maximum transmit power for relaying at UAV is expressed as

$$
P_{r}=\frac{E_{1}+E_{S I}}{(1-\alpha) T}=\frac{\eta \phi_{1}(\theta) \sum_{i=1}^{2}\left|\mathbf{h}_{S R}^{H} \mathbf{w}_{i}\right|^{2} \alpha}{1-\alpha}+\eta \sum_{i=1}^{2}\left|\mathbf{h}_{r r}^{H} \mathbf{s}_{i}\right|^{2}
$$

The received SINR to detect the signal $x_{2}(n)$ and $x_{1}(n)$ at the UAV are respectively written as

$$
\begin{aligned}
& \gamma_{2}^{\mathrm{R}}=\frac{\phi_{1}(\theta)\left|\mathbf{h}_{S R}^{H} \mathbf{w}_{2}\right|^{2}}{\phi_{1}(\theta)\left|\mathbf{h}_{S R}^{H} \mathbf{w}_{1}\right|^{2}+\rho \sum_{i=1}^{2}\left|\mathbf{h}_{r r}^{H} \mathbf{s}_{i}\right|^{2}+\sigma_{r}^{2}}, \\
& \gamma_{1}^{\mathrm{R}}=\frac{\phi_{1}(\theta)\left|\mathbf{h}_{S R}^{H} \mathbf{w}_{1}\right|^{2}}{\rho\left(\left|\mathbf{h}_{r r}^{H} \mathbf{s}_{1}\right|^{2}+\left|\mathbf{h}_{r r}^{H} \mathbf{s}_{2}\right|^{2}\right)+\sigma_{r}^{2}}
\end{aligned}
$$

The received SINR to detect the signal $s_{2}(n)$ at the U1 is expressed as

$$
\gamma_{2}^{\mathrm{U} 1}=\frac{\phi_{2}(\theta)\left|\mathbf{h}_{R 1}^{H} \mathbf{s}_{2}\right|^{2}}{\phi_{2}(\theta)\left|\mathbf{h}_{R 1}^{H} \mathbf{s}_{1}\right|^{2}+\sigma_{1}^{2}}
$$

The received signal to detect its own signal at $\mathrm{U} 1$ is represented by

$$
\gamma_{1}^{\mathrm{U} 1}=\frac{\phi_{2}(\theta)\left|\mathbf{h}_{R 1}^{H} \mathbf{s}_{1}\right|^{2}}{\sigma_{1}^{2}}
$$

The received signal to detect the message $s_{2}(n)$ at $\mathrm{U} 2$ is expressed as

$$
\gamma_{2}^{\mathrm{U} 2}=\frac{\phi_{3}(\theta)\left|\mathbf{h}_{R 2}^{H} \mathbf{s}_{2}\right|^{2}}{\phi_{3}(\theta)\left|\mathbf{h}_{R 2}^{H} \mathbf{s}_{1}\right|^{2}+\sigma_{2}^{2}}
$$

Hence, the available rate of U1 can be written as

$$
R 1=\min \left\{\ln \left(1+\gamma_{1}^{\mathrm{R}}\right), \ln \left(1+\gamma_{1}^{\mathrm{U} 1}\right)\right\} .
$$

The available rate of $\mathrm{U} 2$ can be written as

$R 2=\min \left\{\ln \left(1+\gamma_{2}^{\mathrm{R}}\right), \ln \left(1+\gamma_{2}^{\mathrm{U} 1}\right), \ln \left(1+\gamma_{2}^{\mathrm{U} 2}\right)\right\}$.

According to [46], the sum data rate of the whole system can be calculated as

$$
R_{\text {sum }}=R 1+R 2 \text {. }
$$

\subsection{Problem formulation}

\subsubsection{Sum data rate maximization}

Given the trajectory of UAV, we aim at maximizing sum throughput of system by the joint optimization of four beamforming vectors $\left(\mathbf{w}_{1}, \mathbf{w}_{2}, \mathbf{s}_{1}, \mathbf{s}_{2}\right)$ and time allocation coefficient $\alpha$ in this paper. The problem can be formulated as

$$
\begin{array}{cl}
\max _{\left\{\mathbf{w}_{i}\right\}_{i=1}^{2},\left\{s_{j}\right\}_{j=1}^{2}, \alpha} & R_{\text {sum }} \\
\text { s.t. } \quad & R_{1} \geq \bar{R}, R_{2} \geq \bar{R}, \\
& \left\|\mathbf{w}_{1}\right\|^{2}+\left\|\mathbf{w}_{2}\right\|^{2} \leq P_{s}, \\
& \left\|\mathbf{s}_{1}\right\|^{2}+\left\|\mathbf{s}_{2}\right\|^{2} \leq P_{r}, \\
& 0<\alpha<1,
\end{array}
$$

where the target data rate is set for two NOMA users in constraint (20b); constraint (20c) represents the power constraint at the BS, i.e., the transmission power should not be above the maximum $P_{s}$; constraint (20d) denotes that transmit power at UAV completely depends on the harvested power $P_{r}$; the range of time allocation ratio is given by constraint (20e).

\subsubsection{Harvested energy maximization}

Under QoS requirement of two NOMA users as well as power constraints at the BS and the relay, the harvested energy maximization can be written as

$$
\begin{aligned}
& \max _{\left\{\mathbf{w}_{i}\right\}_{i=1}^{2},\left\{s_{j}\right\}_{j=1}^{2}, \alpha} E_{1}+E_{S I} \\
& \text { s.t. } \quad(20 \mathrm{~b})-(20 \mathrm{e})
\end{aligned}
$$

\section{The proposed beamforming design}

\subsection{Sum data rate maximization}

By simple transformation, the problem (20) can be rewritten as

$$
\max _{\left\{\mathbf{w}_{i}\right\}_{i=1}^{2},\left\{s_{j}\right\}_{j=1}^{2}, \alpha} R_{\text {sum }}
$$

$$
\text { s.t. } \quad \min \left\{\frac{\phi_{2}(\theta)\left|\mathbf{h}_{R 1}^{H} \mathbf{s}_{1}\right|^{2}}{\sigma_{1}^{2}}, \frac{\phi_{1}(\theta)\left|\mathbf{h}_{S R}^{H} \mathbf{w}_{1}\right|^{2}}{\varphi_{1}\left(\mathbf{s}_{1}, \mathbf{s}_{2}\right)}\right\} \geq \bar{\gamma},
$$


$\min \left\{\frac{\phi_{1}(\theta)\left|\mathbf{h}_{S R}^{H} \mathbf{w}_{2}\right|^{2}}{\varphi_{2}\left(\mathbf{w}_{1}, \mathbf{s}_{1}, \mathbf{s}_{2}\right)}, \frac{\phi_{2}(\theta)\left|\mathbf{h}_{R 1}^{H} \mathbf{s}_{2}\right|^{2}}{\varphi_{3}\left(\mathbf{s}_{1}\right)}, \frac{\phi_{3}(\theta)\left|\mathbf{h}_{R 2}^{H} \mathbf{s}_{2}\right|^{2}}{\varphi_{4}\left(\mathbf{s}_{1}\right)}\right\} \geq \bar{\gamma}$

$\frac{\left\|\mathbf{s}_{1}\right\|^{2}+\left\|\mathbf{s}_{2}\right\|^{2}}{\alpha} \leq \frac{\eta_{1} \phi_{1}(\theta) \sum_{i=1}^{2}\left|\mathbf{h}_{S R}^{H} \mathbf{w}_{i}\right|^{2}}{1-\alpha}+\frac{\eta_{2} \sum_{j=1}^{2}\left|\mathbf{h}_{r r}^{H} \mathbf{s}_{j}\right|^{2}}{\alpha}$,

(20c), (20e)

where $\varphi_{2}\left(\mathbf{w}_{1}, \mathbf{s}_{1}, \mathbf{s}_{2}\right)=\phi_{1}(\theta)\left|\mathbf{h}_{S R}^{H} \mathbf{w}_{1}\right|^{2}+$ $\rho P_{r}\left(\left|\mathbf{h}_{r r}^{H} \mathbf{s}_{1}\right|^{2}+\left|\mathbf{h}_{r r}^{H} \mathbf{s}_{2}\right|^{2}\right)+\sigma_{r}^{2}, \quad \varphi_{1}\left(\mathbf{s}_{1}, \mathbf{s}_{2}\right)=$ $\rho P_{r}\left(\left|\mathbf{h}_{r r}^{H} \mathbf{s}_{1}\right|^{2}+\left|\mathbf{h}_{r r}^{H} \mathbf{s}_{2}\right|^{2}\right)+\sigma_{r}^{2}, \varphi_{3}\left(\mathbf{s}_{1}\right)=\phi_{2}(\theta)\left|\mathbf{h}_{R 1}^{H} \mathbf{s}_{1}\right|^{2}+$ $\sigma_{1}^{2}, \varphi_{4}\left(\mathbf{s}_{1}\right)=\phi_{3}(\theta)\left|\mathbf{h}_{R 2}^{H} \mathbf{s}_{1}\right|^{2}+\sigma_{2}^{2}$ and $\bar{\gamma}=\mathrm{e}^{\bar{R}}-1$.

Apparently, the constraint (22e) is convex. However, the formulated problem is highly non-convex due to the non-convex objective (22a) and constraints (22b)-(22d). Next, let us cope with non-convex terms by using inner approximation method.

According to the inequality (45), five non-convex terms of the objective and two non-convex terms of the right side of (22b) can be bounded respectively around $\begin{aligned}\left(\left\{\mathbf{w}_{i}^{k}\right\}_{i=1}^{2},\left\{s_{j}^{k}\right\}_{j=1}^{2}, \alpha^{k}\right) \text { by } & \\ \frac{\phi_{2}(\theta)\left|\mathbf{h}_{R 1}^{H} \mathbf{s}_{1}\right|^{2}}{\sigma_{1}^{2}} & \geq \frac{2 \phi_{2}(\theta) R\left\{\left(\mathbf{h}_{R 1}^{H} \mathbf{s}_{1}^{k}\right)^{*} \mathbf{h}_{R 1}^{H} \mathbf{s}_{1}\right\}}{\sigma_{1}^{2}} \\ & -\frac{\phi_{2}(\theta)\left|\mathbf{h}_{R 1}^{H} \mathbf{s}_{1}^{k}\right|^{2}}{\sigma_{1}^{2}}=\lambda_{1}\left(\mathbf{s}_{1}\right) \\ \frac{\phi_{1}(\theta)\left|\mathbf{h}_{S R}^{H} \mathbf{w}_{1}\right|^{2}}{\varphi_{1}\left(\mathbf{w}_{1}, \mathbf{s}_{1}, \mathbf{s}_{2}\right)} & \geq \frac{2 \phi_{1}(\theta) R\left\{\left(\mathbf{h}_{S R}^{H} \mathbf{w}_{1}^{k}\right)^{*} \mathbf{h}_{S R}^{H} \mathbf{w}_{2}\right\}}{\varphi_{1}\left(\mathbf{w}_{1}^{k}, \mathbf{s}_{1}^{k}, \mathbf{s}_{2}^{k}\right)} \\ & -\frac{\left|\mathbf{h}_{S R}^{H} \mathbf{w}_{2}^{k}\right|^{2}}{\left(\varphi_{1}\left(\mathbf{w}_{1}^{k}, \mathbf{s}_{1}^{k}, \mathbf{s}_{2}^{k}\right)\right)^{2}} \varphi_{1}\left(\mathbf{w}_{1}, \mathbf{s}_{1}, \mathbf{s}_{2}\right) \\ = & \lambda_{2}\left(\left\{\mathbf{w}_{i}\right\}_{i=1}^{2},\left\{s_{j}\right\}_{j=1}^{2}\right)\end{aligned}$

$$
\begin{aligned}
\frac{\phi_{1}(\theta)\left|\mathbf{h}_{S R}^{H} \mathbf{w}_{1}\right|^{2}}{\sigma_{1}^{2}} & \geq \frac{2 \phi_{1}(\theta) R\left\{\left(\mathbf{h}_{S R}^{H} \mathbf{w}_{1}^{k}\right)^{*} \mathbf{h}_{S R}^{H} \mathbf{w}_{1}\right\}}{\sigma_{1}^{2}} \\
& -\frac{\left|\mathbf{h}_{S R}^{H} \mathbf{w}_{1}^{k}\right|^{2}}{\sigma_{1}^{2}}=\lambda_{3}\left(\mathbf{w}_{1}\right)
\end{aligned}
$$

$$
\min \left\{\lambda_{2}\left(\left\{\mathbf{w}_{i}\right\}_{i=1}^{2},\left\{s_{j}\right\}_{j=1}^{2}\right), \chi_{4}\left(\left\{\mathbf{s}_{j}^{k}\right\}_{j=1}^{2}\right), \chi_{5}\left(\left\{\mathbf{s}_{j}\right\}_{j=1}^{2}\right)\right\} \geq \bar{\gamma}
$$

Then, using the inequality (47), $\ln \left(1+\gamma_{1}^{\mathrm{R}}\right)$, $\ln \left(1+\gamma_{1}^{\mathrm{U} 1}\right), \ln \left(1+\gamma_{2}^{\mathrm{R}}\right), \ln \left(1+\gamma_{2}^{\mathrm{U} 1}\right), \ln \left(1+\gamma_{2}^{\mathrm{U} 2}\right)$ of the objective can be innerly approximated by

$$
\begin{aligned}
\ln \left(1+\gamma_{1}^{\mathrm{R}}\right) & \geq \ln \left(1+\frac{\phi_{1}(\theta)\left|\mathbf{h}_{S R}^{H} \mathbf{w}_{1}^{k}\right|^{2}}{\sigma_{1}^{2}}\right)-\frac{\phi_{1}(\theta)\left|\mathbf{h}_{S R}^{H} \mathbf{w}_{1}^{k}\right|^{2}}{\sigma_{1}^{2}} \\
& +\frac{2 \phi_{1}(\theta) R\left\{\left(\mathbf{h}_{S R}^{H} \mathbf{w}_{1}^{k}\right)^{*} \mathbf{h}_{S R}^{H} \mathbf{w}_{1}\right\}}{\sigma_{1}^{2}} \\
& -\frac{\left|\mathbf{h}_{S R}^{H} \mathbf{w}_{1}^{k}\right|^{2}\left(\left|\mathbf{h}_{S R}^{H} \mathbf{w}_{1}^{k}\right|^{2}+\sigma_{1}^{2}\right)}{\sigma_{1}^{2}\left(\sigma_{1}^{2}+\left|\mathbf{h}_{S R}^{H} \mathbf{w}_{1}^{k}\right|^{2}\right)}=\Omega_{1}\left(\mathbf{w}_{1}\right)
\end{aligned}
$$




$$
\begin{aligned}
& \ln \left(1+\gamma_{1}^{\mathrm{U} 1}\right) \geq \ln \left(1+\frac{\phi_{1}(\theta)\left|\mathbf{h}_{R 1}^{H} \mathbf{s}_{1}^{k}\right|^{2}}{\sigma_{1}^{2}}\right)-\frac{\phi_{1}(\theta)\left|\mathbf{h}_{R 1}^{H} \mathbf{s}_{1}^{k}\right|^{2}}{\sigma_{1}^{2}} \\
& +\frac{2 \phi_{1}(\theta) R\left\{\left(\mathbf{h}_{R \mathbf{1}}^{H} \mathbf{s}_{1}^{k}\right)^{*} \mathbf{h}_{R 1}^{H} \mathbf{s}_{1}\right\}}{\sigma_{1}^{2}} \\
& -\frac{\left|\mathbf{h}_{R 1}^{H} \mathbf{s}_{1}^{k}\right|^{2}\left(\left|\mathbf{h}_{R 1}^{H} \mathbf{s}_{1}^{k}\right|^{2}+\sigma_{1}^{2}\right)}{\sigma_{1}^{2}\left(\sigma_{1}^{2}+\left|\mathbf{h}_{R 1}^{H} \mathbf{s}_{1}^{k}\right|^{2}\right)}=\Omega_{2}\left(\mathbf{s}_{1}\right) \\
& \ln \left(1+\gamma_{2}^{\mathrm{R}}\right) \geq \ln \left(1+\frac{\phi_{1}(\theta)\left|\mathbf{h}_{S R}^{H} \mathbf{w}_{2}^{k}\right|^{2}}{\varphi_{1}\left(\mathbf{w}_{1}^{k}, \mathbf{s}_{1}^{k}, \mathbf{s}_{2}^{k}\right)}\right)-\frac{\left|\mathbf{h}_{S R}^{H} \mathbf{w}_{2}^{k}\right|^{2}}{\varphi_{1}\left(\mathbf{w}_{1}^{k}, \mathbf{s}_{1}^{k}, \mathbf{s}_{2}^{k}\right)} \\
& +\frac{2 \phi_{1}(\theta) R\left\{\left(\mathbf{h}_{S R}^{H} \mathbf{w}_{2}^{k}\right)^{*} \mathbf{h}_{S R}^{H} \mathbf{w}_{2}\right\}}{\varphi_{1}\left(\mathbf{w}_{1}^{k}, \mathbf{s}_{1}^{k}, \mathbf{s}_{2}^{k}\right)} \\
& -\frac{\left|\mathbf{h}_{S R}^{H} \mathbf{w}_{2}^{k}\right|^{2} \varphi_{1}\left(\mathbf{w}_{1}, \mathbf{s}_{1}, \mathbf{s}_{2}\right)}{\varphi_{1}\left(\mathbf{w}_{1}^{k}, \mathbf{s}_{1}^{k}, \mathbf{s}_{2}^{k}\right)\left(\varphi_{1}\left(\mathbf{w}_{1}^{k}, \mathbf{s}_{1}^{k}, \mathbf{s}_{2}^{k}\right)+\left|\mathbf{h}_{S R}^{H} \mathbf{w}_{2}^{k}\right|^{2}\right)} \\
& =\Omega_{3}\left(\left\{\mathbf{w}_{i}\right\}_{i=1}^{2},\left\{\mathbf{s}_{j}\right\}_{j=1}^{2}\right) \\
& \ln \left(1+\gamma_{2}^{\mathrm{U} 1}\right) \geq \ln \left(1+\frac{\phi_{1}(\theta)\left|\mathbf{h}_{R 1}^{H} \mathbf{s}_{2}^{k}\right|^{2}}{\varphi_{2}\left(\mathbf{s}_{1}^{k}, \mathbf{s}_{2}^{k}\right)}\right)-\frac{\left|\mathbf{h}_{R 1}^{H} \mathbf{s}_{2}^{k}\right|^{2}}{\varphi_{2}\left(\mathbf{s}_{1}^{k}, \mathbf{s}_{2}^{k}\right)} \\
& +\frac{2 \phi_{2}(\theta) R\left\{\left(\mathbf{h}_{R 1}^{H} \mathbf{s}_{2}^{k}\right)^{*} \mathbf{h}_{R 1}^{H} \mathbf{s}_{2}\right\}}{\varphi_{2}\left(\mathbf{s}_{1}^{k}, \mathbf{s}_{2}^{k}\right)} \\
& -\frac{\left|\mathbf{h}_{R 1}^{H} \mathbf{s}_{2}^{k}\right|^{2} \varphi_{2}\left(\mathbf{s}_{1}, \mathbf{s}_{2}\right)}{\varphi_{2}\left(\mathbf{s}_{1}^{k}, \mathbf{s}_{2}^{k}\right)\left(\varphi_{2}\left(\mathbf{s}_{1}^{k}, \mathbf{s}_{2}^{k}\right)+\left|\mathbf{h}_{R 1}^{H} \mathbf{s}_{2}^{k}\right|^{2}\right)} \\
& =\Omega_{4}\left(\left\{\mathbf{s}_{j}\right\}_{j=1}^{2}\right)
\end{aligned}
$$

$$
\begin{aligned}
\ln \left(1+\gamma_{2}^{\mathrm{U} 2}\right) & \geq \ln \left(1+\frac{\phi_{1}(\theta)\left|\mathbf{h}_{R 2}^{H} \mathbf{s}_{2}^{k}\right|^{2}}{\varphi_{4}\left(\mathbf{s}_{1}^{k}\right)}\right)-\frac{\left|\mathbf{h}_{R 2}^{H} \mathbf{s}_{2}^{k}\right|^{2}}{\varphi_{4}\left(\mathbf{s}_{1}^{k}\right)} \\
& +\frac{2 \phi_{2}(\theta) R\left\{\left(\mathbf{h}_{R 2}^{H} \mathbf{s}_{2}^{k}\right)^{*} \mathbf{h}_{R 2}^{H} \mathbf{s}_{2}\right\}}{\varphi_{4}\left(\mathbf{s}_{1}^{k}\right)} \\
& -\frac{\left|\mathbf{h}_{R 2}^{H} \mathbf{s}_{2}^{k}\right|^{2} \varphi_{4}\left(\mathbf{s}_{1}\right)}{\varphi_{4}\left(\mathbf{s}_{1}^{k}\right)\left(\varphi_{4}\left(\mathbf{s}_{1}^{k}\right)+\left|\mathbf{h}_{R 2}^{H} \mathbf{s}_{2}^{k}\right|^{2}\right)}=\Omega_{5}\left(\left\{\mathbf{s}_{j}\right\}_{j=1}^{2}\right)
\end{aligned}
$$

For $k$ th iteration, the feasible points of the original problem can be generated by solving the convex problem

$$
\begin{gathered}
\max _{\left\{\mathbf{w}_{i}\right\}_{i=1}^{2},\left\{s_{j}\right\}_{j=1}^{2}, \alpha} f_{k}\left(\left\{\mathbf{w}_{i}\right\}_{i=1}^{2},\left\{s_{j}\right\}_{j=1}^{2}, \alpha\right) \\
\text { s.t. } \quad(22 \mathrm{e}),(29)-(31)
\end{gathered}
$$

where $\quad f_{k}\left(\left\{\mathbf{w}_{i}\right\}_{i=1}^{2},\left\{\mathbf{s}_{j}\right\}_{j=1}^{2}, \alpha\right)=\Omega_{1}\left(\mathbf{w}_{1}\right)+\Omega_{2}\left(\mathbf{s}_{1}\right)+\Omega_{3}$ $\left(\left\{\mathbf{w}_{i}\right\}_{i=1}^{2},\left\{\mathbf{s}_{j}\right\}_{j=1}^{2}\right)+\Omega_{4}\left(\left\{\mathbf{s}_{j}\right\}_{j=1}^{2}\right)+\Omega_{5}\left(\left\{\mathbf{s}_{j}\right\}_{j=1}^{2}\right)$ is the approximation of the objective of original problem.

Algorithm 1 Sum throughout Maximization Iteration Algorithm

1: Initilization: Set $n=0$,given maximum tolerance $\varepsilon$ and and generate initial feasible point $\left(\left\{\mathbf{w}_{i}^{0}\right\}_{i=1}^{2},\left\{s_{j}^{0}\right\}_{j=1}^{2}, \alpha^{0}\right)$ by solving (38)

2: Repeat:

3: For given feasible point, obtain optimal solutions $\left(\left\{\mathbf{w}_{i}^{*}\right\}_{i=1}^{2},\left\{s_{j}^{*}\right\}_{j=1}^{2}, \alpha^{*}\right)$ by solving (37)

4: Update: $\mathbf{w}_{1}^{k+1}=\mathbf{w}_{1}^{*}, \mathbf{w}_{2}^{k+1}=\mathbf{w}_{2}^{*}, \mathbf{s}_{1}^{k+1}=\mathbf{s}_{1}^{*}, \mathbf{s}_{2}^{k+1}=$ $\mathbf{s}_{2}^{*}, \alpha^{k+1}=\alpha^{*}$

5: Set $k=k+1$

6: Until: $\left|\left(f_{k+1}-f_{k}\right) / f_{k}\right| \leq \varepsilon$

7: Output: $\mathbf{w}_{1}^{(k)}, \mathbf{w}_{2}^{(k)}, \mathbf{r}_{1}^{(k)}, \mathbf{r}_{2}^{(k)}$

Convergence analysis: The convergence performance of the proposed scheme can be presented in the following proposition.

Proposition 1 Algorithm 1 can generate the feasible points by iteration to make the objective value of (22) become bigger and finally converge to the Karush-KuhnTucker point of (22) after finitely many iterations.

Proof Refer to Appendix 5 for the detailed proof.

Complexity analysis: The computational cost of problem (22) at each iteration is $O\left(m^{2} n^{2.5}+n^{3.5}\right)$, where the optimization problem (22) involves $m=2 M+2 N+1$ the scalar real variables and $n=4$ quadratic and linear constraints [47].

Given the initial point $\left(\left\{\mathbf{w}_{i}^{0}\right\}_{i=1}^{2},\left\{s_{j}^{0}\right\}_{j=1}^{2}, \alpha^{0}\right)$, we can achieve the feasible point of the problem (22) by iterating the following problem

$$
\begin{aligned}
& \min _{\left\{\mathbf{w}_{i}\right\}_{i=1}^{2},\left\{s_{j}\right\}_{j=1}^{2}} \\
& \left\{\frac{\min \left\{\lambda_{1}\left(\mathbf{s}_{1}\right), \lambda_{3}\left(\mathbf{w}_{1}\right)\right\}}{\bar{\gamma}}, \frac{\min \left\{\lambda_{2}\left(\left\{\mathbf{w}_{i}\right\}_{i=1}^{2},\left\{s_{j}\right\}_{j=1}^{2}\right), \chi_{4}\left(\left\{\mathbf{s}_{j}^{k}\right\}_{j=1}^{2}\right), \chi_{5}\left(\left\{\mathbf{s}_{j}\right\}_{j=1}^{2}\right)\right\}}{\bar{\gamma}}\right\} \\
& \text { s.t. } \quad(22 \mathrm{e}),(29)
\end{aligned}
$$

until the objective is greater than or equals 1 . 


\subsection{Harvested energy maximization}

For convenient treatment, the problem can be rewritten as

$\max _{\left\{\mathbf{w}_{i}\right\}_{i=1}^{2},\left\{s_{j}\right\}_{j=1}^{2}, \alpha} \eta_{1} f_{1}(\theta) \sum_{i=1}^{2}\left|\mathbf{h}_{S R}^{H} \mathbf{w}_{i}\right|^{2} \alpha+\eta_{2} f_{1}(\theta) \sum_{j=1}^{2}\left|\mathbf{h}_{r r}^{H} \mathbf{s}_{j}\right|^{2}(1-\alpha)$

$$
\text { s.t. } \quad(22 \mathrm{~b})-(22 \mathrm{e}) \text {, }
$$

By introducing auxiliary variable $\lambda_{1}, \lambda_{2}$, we have

$$
\alpha \lambda_{1} \leq 1 \text { and }(1-\alpha) \lambda_{2} \leq 1
$$

Hence, the problem (39) can be expressed equivalently as

$$
\max _{\left\{\mathbf{w}_{i}\right\}_{i=1}^{2},\left\{s_{j}\right\}_{j=1}^{2}, \alpha, \lambda_{1}, \lambda_{2}} \frac{\eta_{1} f_{1}(\theta) \sum_{i=1}^{2}\left|\mathbf{h}_{S R}^{H} \mathbf{w}_{i}\right|^{2} \alpha}{\lambda_{1}}+\frac{\eta_{1} f_{1}(\theta) \sum_{j=1}^{2}\left|\mathbf{h}_{r r}^{H} \mathbf{s}_{j}\right|^{2}(1-\alpha)}{\lambda_{2}}
$$

$$
\text { s.t. } \quad(22 \mathrm{~b})-(22 \mathrm{~d})
$$

$$
\text { (22e), (40). }
$$

It is easily found that the constraints (41c) are convex. As for non-convex constraints (41b), we can approximate these into convex ones by referring to sum throughput maximization part, which can be expressed as (29)-(31).

Next, let us cope with the non-convex objective using (41a). Two terms of the objective can be bounded respectively by

$$
\begin{aligned}
\frac{\eta_{1} f_{1}(\theta) \sum_{i=1}^{2}\left|\mathbf{h}_{S R}^{H} \mathbf{w}_{i}\right|^{2}}{\lambda_{1}} & \geq \eta_{1} f_{1}(\theta) \sum_{i=1}^{2}\left(\frac{2 R\left\{\left(\mathbf{h}_{S R}^{H} \mathbf{w}_{i}^{k}\right)^{*} \mathbf{h}_{S R}^{H} \mathbf{w}_{i}\right\}}{\lambda_{1}^{k}}-\frac{\left|\mathbf{h}_{S R}^{H} \mathbf{w}_{i}^{k}\right|^{2} \lambda_{1}}{\left(\lambda_{1}^{k}\right)^{2}}\right) \\
= & \mathrm{g}_{1}\left(\left\{\mathbf{w}_{i}\right\}_{i=1}^{2}, \lambda_{1}\right)
\end{aligned}
$$

$$
\begin{aligned}
\frac{\eta_{2} \sum_{j=1}^{2}\left|\mathbf{h}_{r r}^{H} \mathbf{s}_{j}\right|^{2}}{\lambda_{2}} & \geq \eta_{2} \sum_{j=1}^{2}\left(\frac{2 R\left\{\left(\mathbf{h}_{r r}^{H} \mathbf{s}_{j}^{k}\right)^{*} \mathbf{h}_{r r}^{H} \mathbf{s}_{j}\right\}}{\lambda_{2}^{k}}-\frac{\left|\mathbf{h}_{r r}^{H} \mathbf{s}_{j}^{k}\right|^{2} \lambda_{2}}{\left(\lambda_{2}^{k}\right)^{2}}\right) \\
= & \mathrm{g}_{2}\left(\left\{\mathbf{s}_{j}\right\}_{j=1}^{2}, \lambda_{2}\right)
\end{aligned}
$$

In result, at $k$ th iteration, the feasible point of (41) can be generated by solving the following problem:

$$
\begin{gathered}
\max _{\left\{\mathbf{w}_{i}\right\}_{i=1}^{2},\left\{s_{j}\right\}_{j=1}^{2}, \alpha, \lambda_{1}, \lambda_{2}} g\left(\left\{\mathbf{w}_{i}\right\}_{i=1}^{2},\left\{s_{j}\right\}_{j=1}^{2}, \alpha, \lambda_{1}, \lambda_{2}\right) \\
\text { s.t. } \quad(29)-(31),(41 \mathrm{c}),(42)-(43) .
\end{gathered}
$$

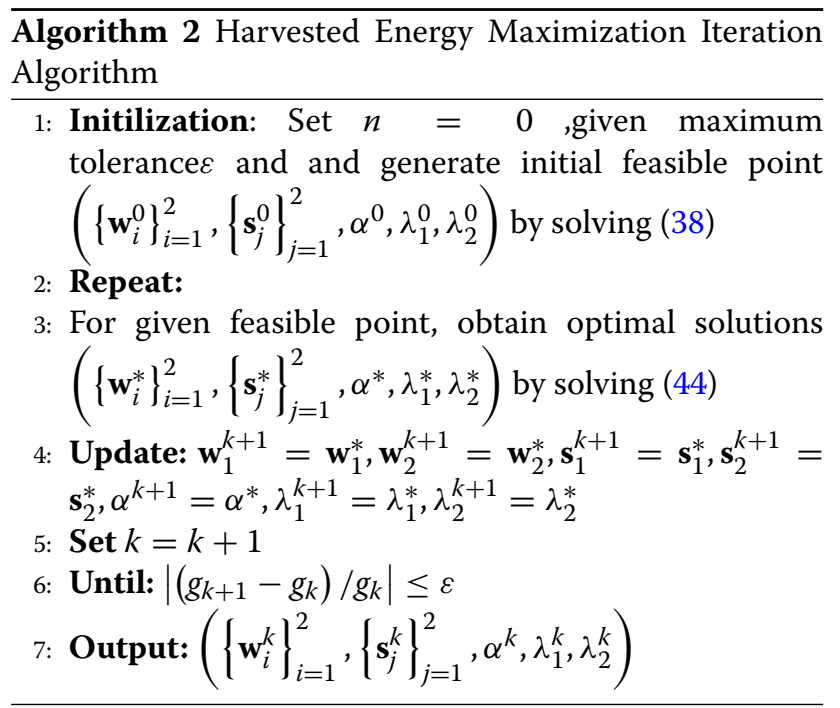

where $g\left(\left\{\mathbf{w}_{i}\right\}_{i=1}^{2},\left\{s_{j}\right\}_{j=1}^{2}, \alpha, \lambda_{1}, \lambda_{2}\right)=\mathrm{g}_{1}\left(\left\{\mathbf{w}_{i}\right\}_{i=1}^{2}, \lambda_{1}\right)+$ $\mathrm{g}_{2}\left(\left\{\mathbf{s}_{j}\right\}_{j=1}^{2}, \lambda_{2}\right)$.

Convergence analysis: Algorithm 2 produces nondecreasing sequence and finally converges to the KKT point of (39) after finitely many iterations, whose proof can refer to Appendix 5.

Complexity analysis: The computational cost of problem (22) at each iteration is $O\left(m^{2} n^{2.5}+n^{3.5}\right)$, where the optimization problem (22) involves $m=2 M+2 N+1$ the scalar real variables and $n=6$ quadratic and linear constraints.

Given the $\lambda_{1}$ and $\lambda_{2}$, the initial feasible point of (41) can be generated by iterating optimization problem (38).

\section{Simulation results}

This section validates the proposed beamforming design for the UAV-enabled cooperative NOMA network by simulation results. Herein, the channel power gain at the reference distance $d_{0}=1 \mathrm{~m}$ is up to $\beta=-65 \mathrm{~dB}$. The other environment coefficients related with channel model are set by $v_{1}=5, v_{2}=-1, v_{2}=-1, v_{4}=42, \mu_{1}=\frac{2}{\pi} \ln 3$ and $\mu_{2}=9$. The distances from all nodes on the ground to central point are assumed to be $d_{s}=50 \mathrm{~m}, d_{1}=50 \mathrm{~m}$, and $d_{2}=65 \mathrm{~m}$. Unless otherwise stated, the default values of other parameters are displayed in Table 1.

For comparison, we present three baseline transmission schemes as below:

- "FD+NOMA+SWIPT with fixed $\alpha=0.5$ ": the only difference between this scheme and the proposed scheme is that the scheme does not take adopts fixed time allocation coefficient $\alpha$.

- "HD+NOMA+SWIPT": different from the proposed scheme, the strategy adopts HD mode. 
Table 1 Default values of other parameters

\begin{tabular}{ll}
\hline Parameter & Value \\
\hline System bandwidth & $1 \mathrm{M}$ \\
Maximum transmit power at BS $\left(P_{S}\right)$ & $20 \mathrm{dBm}$ \\
Noise power $\left(\sigma_{r}^{2}=\sigma_{1}^{2}=\sigma_{2}^{2}\right)\left(P_{0}\right)$ & $-70 \mathrm{dBm}$ \\
The number of transmit antenna at BS and UAV $(M, N)$ & $M=N=4$ \\
SI suppress level $(\rho)$ & $-50 \mathrm{~dB}$ \\
Tolerant error $(\varepsilon)$ & $10^{-6}$ \\
Energy conversion efficiency $(\eta)$ & 0.8 \\
Minimum data rate at U1 and U2 $(\bar{R})$ & $1 \mathrm{Mbits}$ \\
Azimuth angle $(\theta)$ & $45^{\circ}$ \\
The altitude of UAV $(h)$ & $30 \mathrm{~m}$ \\
Circle radius of UAV's fight $(r)$ & $40 \mathrm{~m}$ \\
\hline
\end{tabular}

•"FD+OMA+SWIPT": in this scheme, traditional time division multiple access technique is employed, which separates the bandwidth equally for BS-UAV-U1 and BSUAV-U2, respectively.

\subsection{Convergence behavior}

Figure 3 depicts the behaviors of the proposed Algorithm 1. We can observe that two algorithms can both converge to at least local optimum in fifteen steps, which has demonstrated the superiority of the proposed algorithms.

\subsection{Comparison of schemes}

In Fig. 4, we plot the sum throughput of the system in terms of transmission power at BS $P_{S}$ with fixed time allocation ratio $\alpha=0.5$ and fixed position of UAV, i.e., $(r \cos \theta, \sin \theta, h)$. As expected, the sum throughput

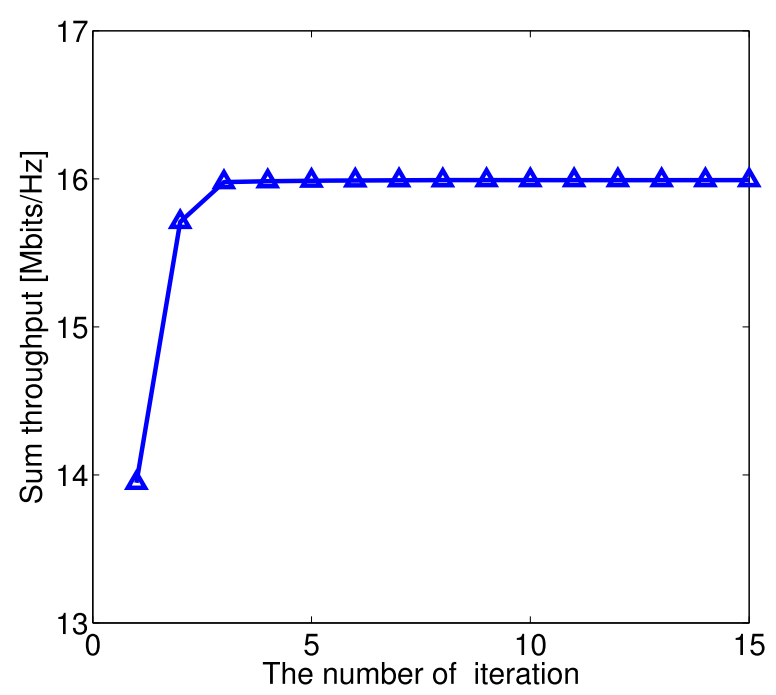

Fig. 3 Convergence of Algorithm 1 of the system increases with $P_{s}$. The proposed scheme yields the best among all schemes. Clearly, FD mode brings more performance gain than the HD one. What is more, it is seen that NOMA schemes can achieve higher spectral efficiency compared to OMA scheme, which can verify the advantage of NOMA in improving spectral efficiency.

Figure 5 shows the impact of azimuth angle $\theta$ of UAV's fight trajectory on the sum throughput of system for two different schemes. Clearly, the throughput performance first increases with $\theta$ and decreases with $\theta$ for the proposed scheme and "FD+OMA+SWIPT" scheme. However, the optimal azimuth angles for two schemes are different. The optimum $\theta$ is located at $90^{\circ}$ in proposed scheme while the optimum $\theta$ is located at $120^{\circ}$ in the "FD+OMA+SWIPT" scheme. In addition, it is observed that the proposed scheme outperforms the "FD+OMA+SWIPT" scheme whatever the value of $\theta$ is, which implied the advantage of using NOMA in improving spectral efficiency.

Figure 6 plots the sum throughput of the system versus time allocation coefficient for different circle radius of UAV's fight. As shown in Fig. 6, the sum throughput of system reaches to peak when the value of time allocation coefficient is up to 0.38 probably. Furthermore, with the decreasing of the circular radius, the sum throughput of system degrades, which demonstrates that the better throughput performance prefers bigger range of UAV's fight.

We also investigated the sum throughput of system versus the UAV's altitude in Fig. 7. As expected, the proposed scheme yields the best among all transmission schemes. Apparently, with the increase of altitude of UAV, the performance gain degrades for all strategies due to bigger channel fading. Additionally, the performance gap becomes smaller between the proposed scheme and two baseline strategies with the increase of altitude of UAV. Therefore, the proposed scheme is more approximate to mid- and low-altitude mountainous region.

Figure 8 illustrates the sum throughput of system versus SI cancelation level. As shown in this figure, the sum throughput of system decreases with the decrease of SI cancelation level in the proposed scheme. Apparently, the performance of the proposed scheme is superior to ones in the HD scheme when $\rho \leq-25 \mathrm{~dB}$, and the performance of the proposed scheme is inferior to ones in HD scheme when $\rho \geq-25 \mathrm{~dB}$.

Finally, we present the harvested energy at UAV versus the azimuth angle $\theta$ of UAV's fight trajectory for the two schemes. Obviously, the harvested energy at UAV is increasingly improved when the UAV is getting closer to the BS (Fig. 9). Moreover, we can find that the proposed scheme outperforms the fixed $\alpha$ scheme, which can imply the importance of dynamically changing $\alpha$. 


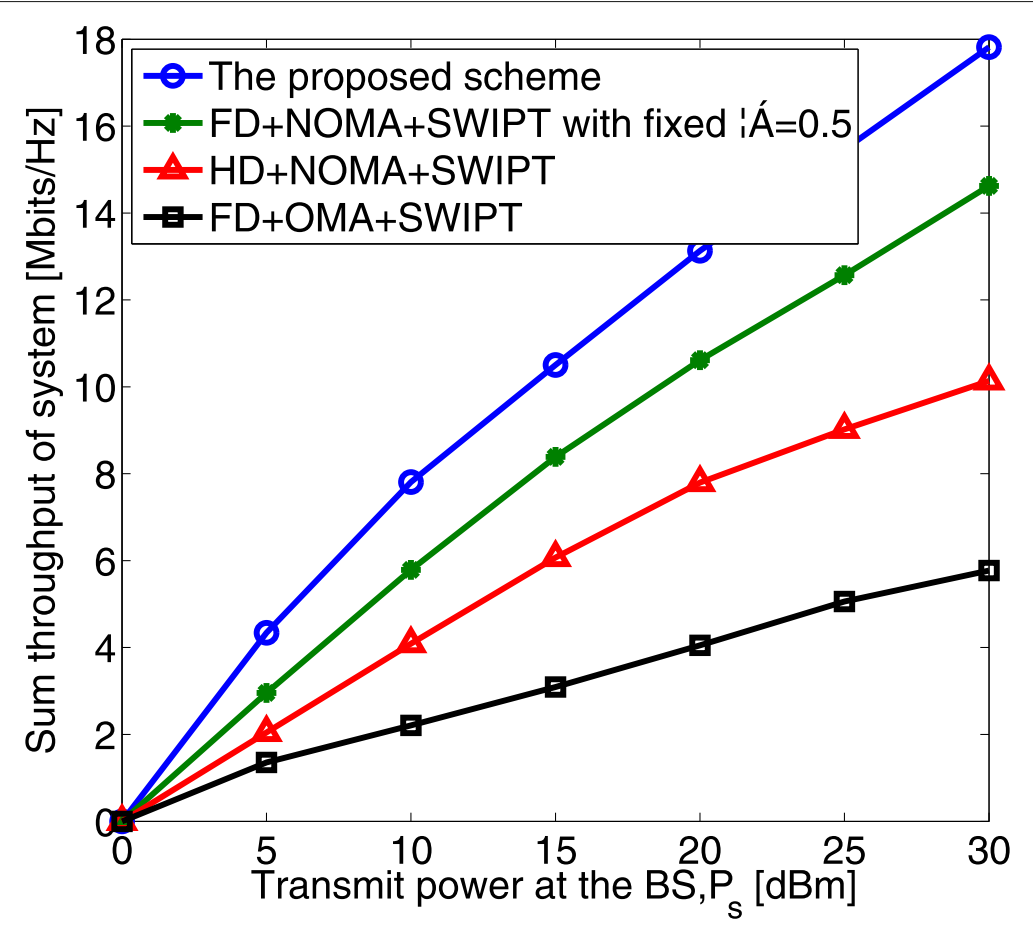

Fig. 4 Sum throughput of system versus transmission power at BS

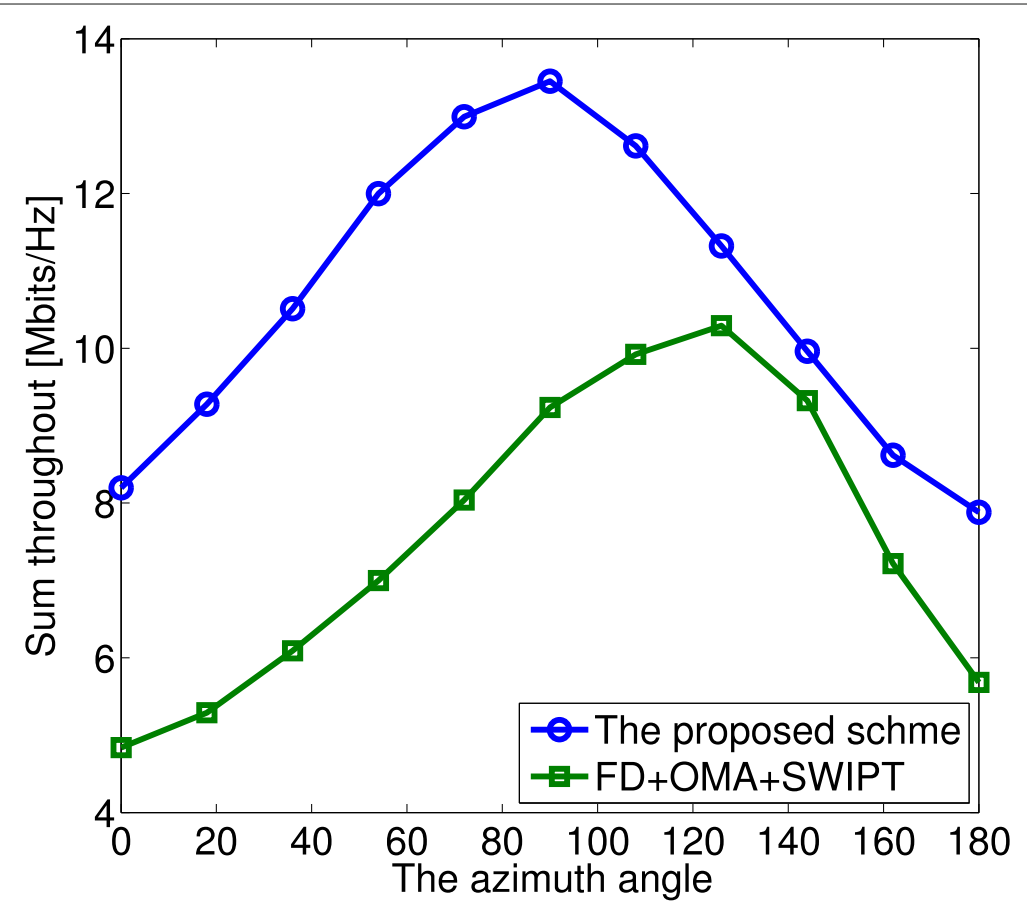

Fig. 5 Sum throughput of system versus azimuth angle 


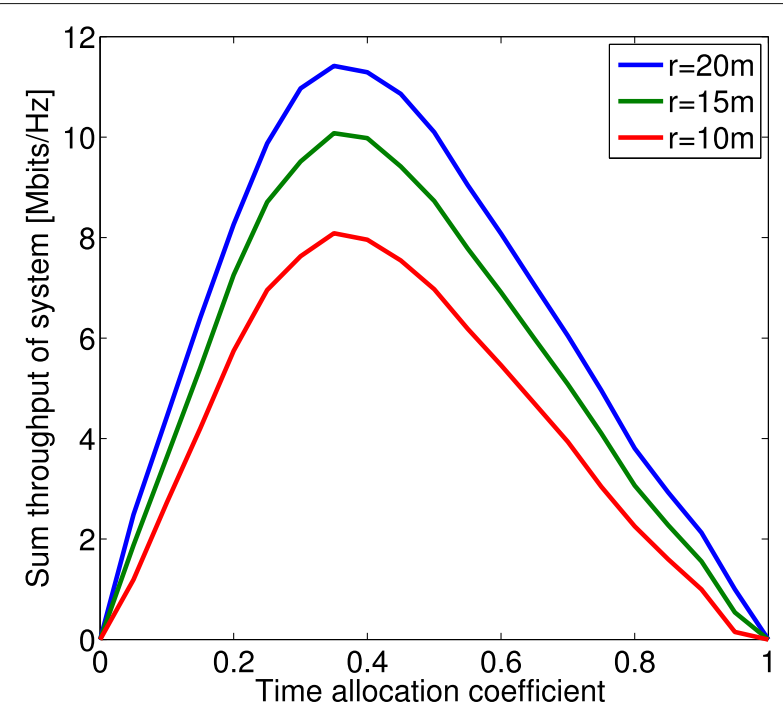

Fig. 6 Sum throughput of system versus time allocation coefficient $\alpha$

time allocation ratio. Numerical results imply that the proposed scheme achieves more performance gain than other strategies.

\section{Appendix 1}

Since $\varphi(x, y)=\frac{x^{2}}{y}$ is convex, thus, it is true that

$$
\frac{x^{2}}{y} \geq 2 \frac{\bar{x}^{*} x}{\bar{y}}-\frac{|\bar{x}|^{2}}{\bar{y}^{2}} y
$$

Following from [39], we have the below inequalities

$$
\begin{aligned}
& \ln \left(1+\frac{|x|^{2}}{y}\right) \geq \ln \left(1+\frac{|\bar{x}|^{2}}{\bar{y}}\right)-\frac{|\bar{x}|^{2}}{\bar{y}}+\frac{2 R\{\bar{x} * x\}}{\bar{y}}-\frac{|\bar{x}|^{2}\left(|\bar{x}|^{2}+y\right)}{\bar{y}\left(\bar{y}+|\bar{x}|^{2}\right)} \\
& \frac{\ln (1+\omega)}{v} \geq \frac{2 \ln (1+\bar{\omega})}{\bar{v}}+\frac{\bar{\omega}}{(\bar{\omega}+1) \bar{v}}-\frac{\bar{\omega}^{2}}{(\bar{\omega}+1) \bar{v}} \frac{1}{\omega}-\frac{\ln (1+\bar{\omega})}{\bar{v}^{2}} v
\end{aligned}
$$

\section{Conclusion}

We have considered a UAV-enabled cooperative NOMA system with application of SWIPT, where the UAV harvests the energy from the BS in the first phase and relay signals in FD mode with the harvested energy. To maximize the sum throughput of system, we have proposed a low-complexity algorithm to solve the joint optimization problem of beamforming vectors and

for all $x \in \mathbb{C}, \bar{x} \in \mathbb{C}, y>0$ and $\bar{y}>0$ and over the feasible region $2 x^{*} x-\left|x^{*}\right|^{2}>0$.

\section{Appendix 2}

It is easily found that

$$
f\left(\left\{\mathbf{w}_{i}\right\}_{i=1}^{2},\left\{\mathbf{s}_{j}\right\}_{j=1}^{2}, \alpha\right) \geq f_{k}\left(\left\{\mathbf{w}_{i}^{k}\right\}_{i=1}^{2},\left\{\mathbf{s}_{j}^{k}\right\}_{j=1}^{2}, \alpha^{k}\right)
$$

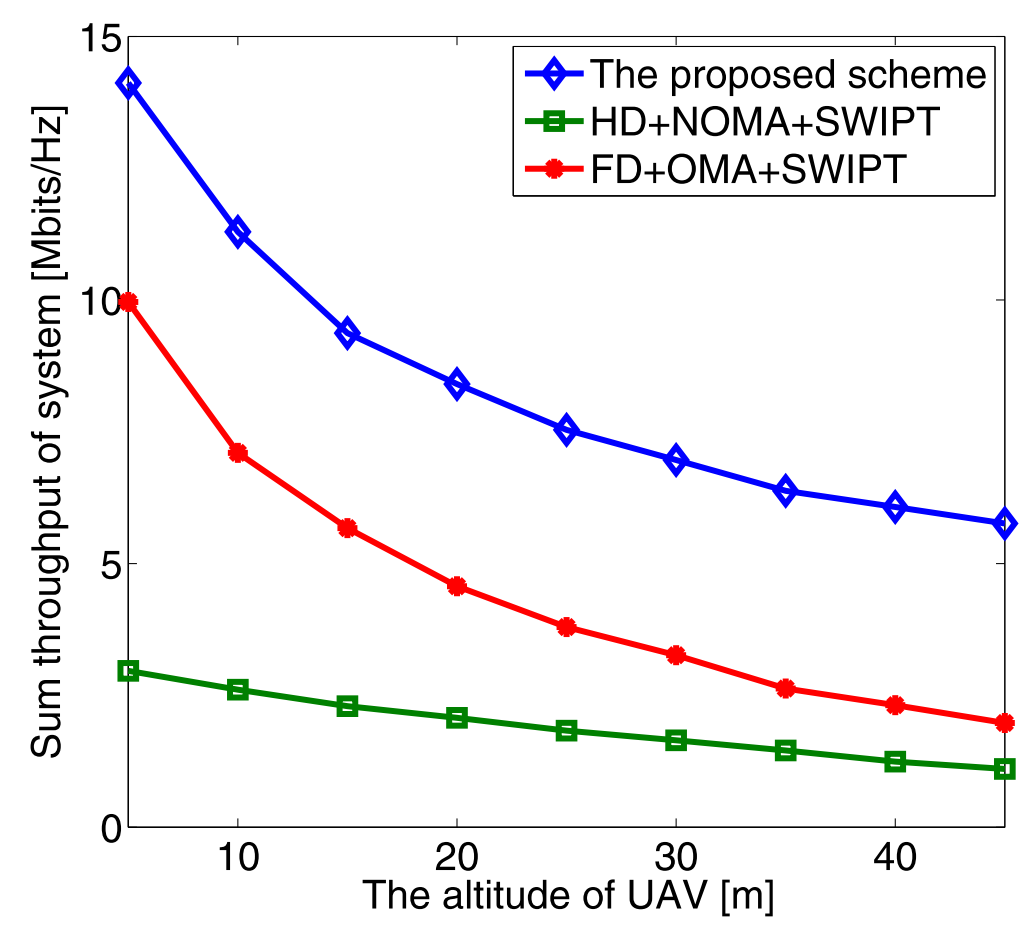

Fig. 7 Sum throughput of system versus the altitude of UAV 


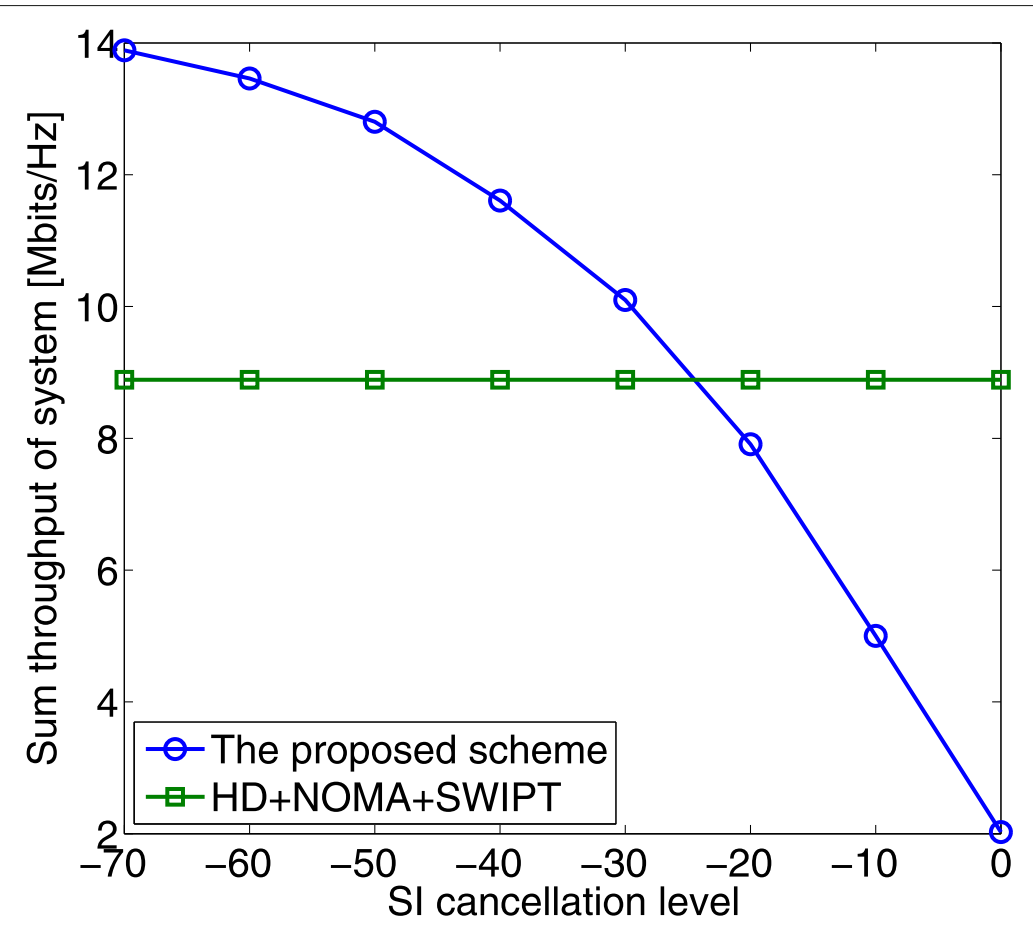

Fig. 8 Sum throughput of system versus SI cancelation level

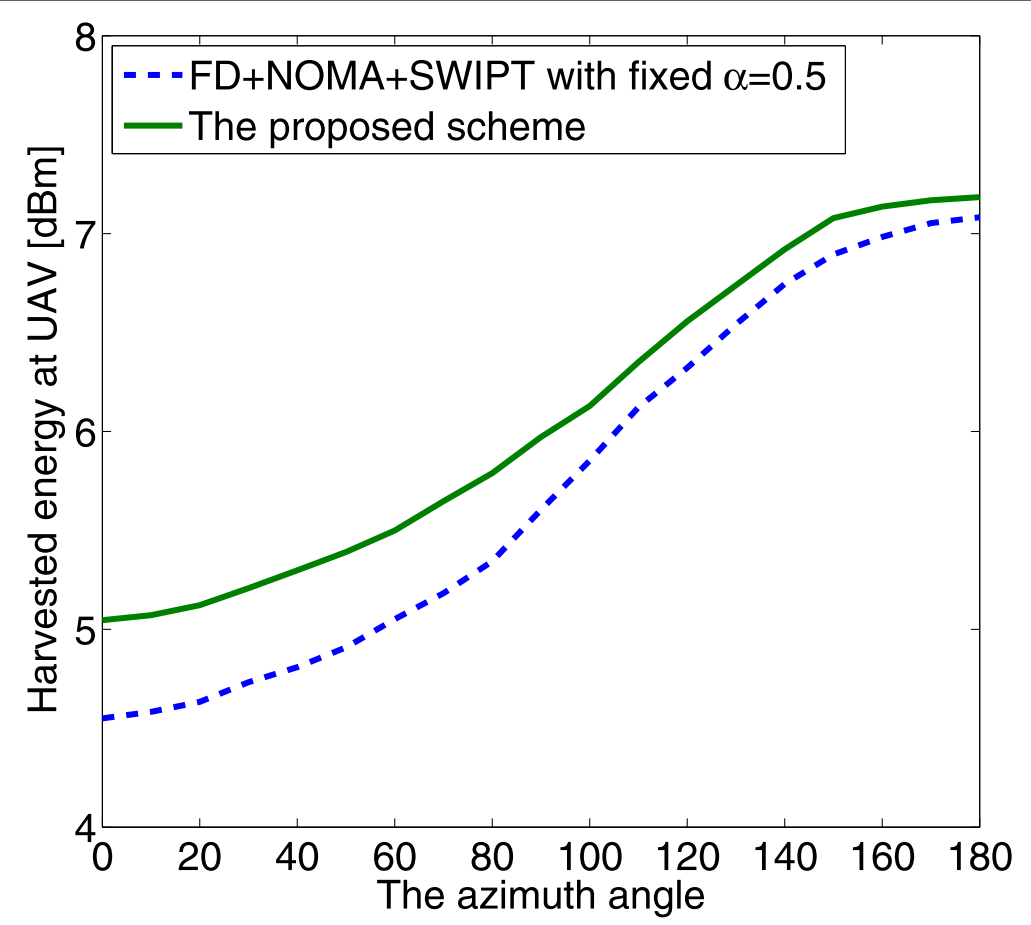

Fig. 9 Harvested energy at UAV versus azimuth angle 
and

$f\left(\left\{\mathbf{w}_{i}^{k}\right\}_{i=1}^{2},\left\{\mathbf{s}_{j}^{k}\right\}_{j=1}^{2}, \alpha^{k}\right)=f_{k}\left(\left\{\mathbf{w}_{i}^{k}\right\}_{i=1}^{2},\left\{\mathbf{s}_{j}^{k}\right\}_{j=1}^{2}, \alpha^{k}\right)$.

Moreover, Algorithm 1 produces non-decreasing sequence provided that $\left(\left\{\mathbf{w}_{i}^{k}\right\}_{i=1}^{2},\left\{\mathbf{s}_{j}^{k}\right\}_{j=1}^{2}, \alpha^{k}\right) \neq$ $\left(\left\{\mathbf{w}_{i}^{k+1}\right\}_{i=1}^{2},\left\{\mathbf{s}_{j}^{k+1}\right\}_{j=1}^{2}, \alpha^{k+1}\right)$, i.e.,

$f\left(\left\{\mathbf{w}_{i}^{k+1}\right\}_{i=1}^{2},\left\{\mathbf{s}_{j}^{k+1}\right\}_{j=1}^{2}, \alpha^{k+1}\right)=f_{k}\left(\left\{\mathbf{w}_{i}^{k}\right\}_{i=1}^{2},\left\{\mathbf{s}_{j}^{k}\right\}_{j=1}^{2}, \alpha^{k}\right)$.

Thus, we can derive that the feasible point generated by Algorithm 1 can make objective value of (22) become bigger due to that

$$
\begin{aligned}
f\left(\left\{\mathbf{w}_{i}^{k+1}\right\}_{i=1}^{2},\left\{\mathbf{s}_{j}^{k+1}\right\}_{j=1}^{2}, \alpha^{k+1}\right) & \geq f_{k}\left(\left\{\mathbf{w}_{i}^{k+1}\right\}_{i=1}^{2},\left\{\mathbf{s}_{j}^{k+1}\right\}_{j=1}^{2}, \alpha^{k+1}\right) \\
& \geq f_{k}\left(\left\{\mathbf{w}_{i}^{k}\right\}_{i=1}^{2},\left\{\mathbf{s}_{j}^{k}\right\}_{j=1}^{2}, \alpha^{k}\right) \\
= & f\left(\left\{\mathbf{w}_{i}^{k}\right\}_{i=1}^{2},\left\{\mathbf{s}_{j}^{k}\right\}_{j=1}^{2}, \alpha^{k}\right)
\end{aligned}
$$

The inequality (51) demonstrated that the feasible point $\left(\left\{\mathbf{w}_{i}^{k+1}\right\}_{i=1}^{2},\left\{s_{j}^{k+1}\right\}_{j=1}^{2}, \alpha^{k+1}\right)$ for $(k+1)$ th iteration is better than the feasible point $\left(\left\{\mathbf{w}_{i}^{k}\right\}_{i=1}^{2},\left\{s_{j}^{k}\right\}_{j=1}^{2}, \alpha^{k}\right)$ for $k$ th iteration. According to Cauchy's theorem, the bounded sequence $\left(\left\{\mathbf{w}_{i}^{k}\right\}_{i=1}^{2},\left\{s_{j}^{k}\right\}_{j=1}^{2}, \alpha^{k}\right)$ would converge to the limited point $\left(\left\{\overline{\mathbf{w}}_{i}\right\}_{i=1}^{2},\left\{\bar{s}_{j}\right\}_{j=1}^{2}, \bar{\alpha}\right)$, i.e.,

$\lim _{v \rightarrow \infty}\left[f\left(\left\{\mathbf{w}_{i}^{k_{v}}\right\}_{i=1}^{2},\left\{s_{j}^{k_{v}}\right\}_{j=1}^{2}, \alpha^{k_{v}}\right)-f\left(\left\{\overline{\mathbf{w}}_{i}\right\}_{i=1}^{2},\left\{\bar{s}_{j}\right\}_{j=1}^{2}, \bar{\alpha}^{k}\right)\right]=0$

Motivated by this, we can derive that

$$
\lim _{k \rightarrow \infty} f\left(\left\{\mathbf{w}_{i}^{k}\right\}_{i=1}^{2},\left\{s_{j}^{k}\right\}_{j=1}^{2}, \alpha^{k}\right)=f\left(\left\{\overline{\mathbf{w}}_{i}\right\}_{i=1}^{2},\left\{\bar{s}_{j}\right\}_{j=1}^{2}, \bar{\alpha}\right)
$$

due to that

$$
\begin{aligned}
0= & \lim _{v \rightarrow \infty}\left[f\left(\left\{\mathbf{w}_{i}^{k_{v}}\right\}_{i=1}^{2},\left\{s_{j}^{k_{v}}\right\}_{j=1}^{2}, \alpha^{k_{v}}\right)-f\left(\left\{\overline{\mathbf{w}}_{i}\right\}_{i=1}^{2},\left\{\bar{s}_{j}\right\}_{j=1}^{2}, \bar{\alpha}^{k}\right)\right] \\
& \leq \lim _{k \rightarrow \infty}\left[f\left(\left\{\mathbf{w}_{i}^{k}\right\}_{i=1}^{2},\left\{s_{j}^{k}\right\}_{j=1}^{2}, \alpha^{k}\right)-f\left(\left\{\overline{\mathbf{w}}_{i}\right\}_{i=1}^{2},\left\{\bar{s}_{j}\right\}_{j=1}^{2}, \bar{\alpha}\right)\right] \\
& \leq \lim _{v \rightarrow \infty}\left[f\left(\left\{\mathbf{w}_{i}^{k_{v+1}}\right\}_{i=1}^{2},\left\{s_{j}^{k_{v+1}}\right\}_{j=1}^{2}, \alpha^{k_{v+1}}\right)-f\left(\left\{\overline{\mathbf{w}}_{i}\right\}_{i=1}^{2},\left\{\bar{s}_{j}\right\}_{j=1}^{2}, \bar{\alpha}\right)\right]
\end{aligned}
$$$$
=0 \text {. }
$$

where $k_{v} \leq k \leq k_{v+1}$. Thus, each accumulation point $\left(\left\{\overline{\mathbf{w}}_{i}\right\}_{i=1}^{2},\left\{\bar{s}_{j}\right\}_{j=1}^{2}, \bar{\alpha}\right)$ of the feasible point $\left(\left\{\mathbf{w}_{i}^{k}\right\}_{i=1}^{2},\left\{s_{j}^{k}\right\}_{j=1}^{2}, \alpha^{k}\right)$ generated by Algorithm 1 satisfies the KKT condition [48]. Proposition 1 has been proved.

\section{Abbreviations}

AF: Amplify-and-forward; BS: Base station; CSCG: Circularly symmetric complex Gaussian; CSI: Channel state information; DF: Decode-and-forward; FD: Full-duplex; NOMA: Non-orthogonal multiple access; OMA: Orthogonal multiple access; QoS: Qualty of service; SIC: Successive interference cancelation; SWIPT: Simultaneous wireless information and power transfer; TS: Time-switching; UAV: Unmanned aerial vehicle

\section{Acknowledgements}

Not applicable.

\section{Authors' contributions}

GM is the main author of the current paper. GM contributed to the development of the ideas, design of the study, theory, result analysis, and article writing. GM conceived, designed, and performed the experiments. GM undertook revision works of the paper. The author read and approved the final manuscript.

\section{Funding}

This paper was supported by the Science and Technology Development Program of He'nan Educational Committee(No. 13A510213).

\section{Availability of data and materials}

Data sharing not applicable to this article as no datasets were generated or analyzed during the current study.

\section{Competing interests}

The author declares that there are no competing interests.

Received: 23 November 2019 Accepted: 13 February 2020

Published online: 04 March 2020

\section{References}

1. U. Iqbal, M. S. Sadiq, S. I. A. Shah, in Proc. Int. Conf. Eng. Emerg. Technol (ICEET). Design, development and fabrication of airdrop mechanism for first aid kit drop in unmanned disaster relief helicopter (ICEET, Lahore, 2016), pp. 1-6

2. G. Q. Li, X. G. Zhou, J. Yin, Q. Y. Xiao, in Proc. Int. Arch. Photogramm., Remote Sens. Spatial Inf. Sci. (ISPRS). An UAV scheduling and planning method for post-disaster survey (ISPRS Archives, Toronto, 2014), pp. 169-172

3. Z. Kaleem, M. H. Rehmani, Amateur drone monitoring: state-of-the-art architectures, key enabling technologies, and future research directions. IEEE Wirel. Commun. 25(2), 150-159 (2018)

4. Y. Zeng, R. Zhang, T. J. Lim, Wireless communications with unmanned aerial vehicles: opportunities and challenges, (2016)

5. T. M. Nguyen, W. Ajib, C. Assi, A novel cooperative NOMA for designing UAV-assisted wireless backhaul networks. IEEE J. Sel. Areas Commun. 36(11), 2497-2507 (2018)

6. S. Zhang, H. Zhang, Q. He, K. Bian, L. Song, Joint trajectory and power optimization for UAV relay networks. IEEE Commun. Lett. 22(1), 161164 (2018)

7. J. Kakar, A. Chaaban, V. Marojevic, A. Sezgin, in Proc. IEEE Annual International Symposium on Personal, Indoor, and Mobile Radio Communications (PIMRC), Bologna. UAV-aided multiway communications (IEEE Xplore, Italy, 2018), pp. 1169-1173

8. X. Jiang, Z. Wu, Z. Yin, Z. Yang, Power and trajectory optimization for UAV-enabled amplify-and-forward relay networks. IEEE Access. 6 , 48688-48696 (2018)

9. C. Li, S. Zhang, P. Liu, F. Sun, J. M. Cioffi, L. Yang, Overhearing protocol design exploiting inter-cell interference in cooperative green networks. IEEE Trans. Veh. Technol. 65(1), 441-446 (2016) 
10. Q. Li, W.-K. Ma, Spatially selective artificial-noise aided transmit optimization for MISO multi-eves secrecy rate maximization. IEEE Trans. Sig. Process. 61(10), 2704-2717 (2013)

11. G. Liu, F. R. Yu, H. Ji, In-band full-duplex relaying: a survey, research issues and challenges. IEEE Commun. Surv. Tut. 17(2), 500-524 (2015)

12. Y. Saito, Y. Kishiyama, A. Benjebbour, T. Nakamura, A. Li, K. Higuchi, in Proc. IEEE 77th Veh. Technol. Conf. (VTC Spring). Non-orthogonal multiple access (NOMA) for cellular future radio access, (Dresden, 2013), pp. 1-5

13. Z. Ding, et al., Application of non-orthogonal multiple access in LTE and 5G networks. IEEE Commun. Mag. 55(2), 185-191 (2017)

14. K. M. Rabie, B. Adebisi, M. S. Alouini, Half-duplex and full-duplex AF and DF relaying with energy-harvesting in log-normal fading. IEEE Trans. Green Commun. Netw. 1(4), 468-480 (2017)

15. H. A. Suraweera, I. Krikidis, G. Zheng, C. Yuen, P. J. Smith, Low-complexity end-to-end performance optimization in MIMO full-duplex relay systems. IEEE Trans. Wirel. Commun. 13(2), 913-927 (2014)

16. C. Li, H. J. Yang, F. Sun, J. M. Cioffi, L. Yang, Multiuser overhearing for cooperative two-way multiantenna relays. IEEE Trans. Veh. Technol. 65(5), 3796-3802 (2016)

17. D. P. M. Osorio, E. E. B. Olivo, H. Alves, J. C. S. S. Filho, M. Latva-Aho, Exploiting the direct link in full-duplex amplify-and-forward relaying networks. IEEE Sig. Process. Lett. 22(10), 1766-1770 (2015)

18. B. Yu, L. Yang, X. Cheng, R. Cao, in MILCOM 2013 - 2013 IEEE Military Communications Conference. Relay location optimization for full-duplex decode-and-forward relaying, (2013), pp. 13-18. https://doi.org/10.1109/ milcom.2013.11

19. T. Riihonen, S. Werner, R. Wichman, in Proc. IEEE Conf. Rec. Asilomar Conf. Signals, Syst. Comput. Transmit power optimization for multiantenna decode-and-forward relays with loopback self-interference from full-duplex operation, (Pacific Grove, 2011), pp. 1408-1412. https://doi. org/10.1109/acssc.2011.6190248

20. B. Yu, L. Yang, X. Cheng, R. Cao, Power and location optimization for full-duplex decode-and-forward relaying. IEEE Trans. Commun. 63(12), 4743-4753 (2015)

21. C. Zhong, X. Jiang, F. Qu, Z. Zhang, Multi-antenna wireless legitimate surveillance systems: design and performance analysis. IEEE Trans. Wirel. Commun. 16, 4585-4599 (2017)

22. M. Hua, Y. Wang, Z. Zhang, C. Li, Y. Huang, L. Yang, in China Communications. Outage probability minimization for low-altitude UAV-enabled full-duplex mobile relaying systems, (2018). https://doi.org/ 10.1109/cc.2018.8387983

23. Q. Song, F. Zheng, Y. Zeng, J. Zhang, Joint beamforming and power allocation for UAV-enabled full-duplex relay. IEEE Trans. Veh. Technol. 68(2), 1657-1671 (2019)

24. Z. Ding, X. Lei, G. K. Karagiannidis, R. Schober, J. Yuan, V. Bhargava, A survey on non-orthogonal multiple access for $5 \mathrm{G}$ networks: research challenges and future trends. IEEE J. Sel. Areas Commun. 35(10), 2181-2195 (2017)

25. W. Wu, F. Zhou, P. Li, P. Deng, B. Wang, V. C. M. Leung, in ICC 2019- 2019 IEEE International Conference on Communications (ICC). Energy-efficient secure NOMA-enabled mobile edge computing networks, (Shanghai, 2019), pp. 1-6. https://doi.org/10.1109/icc.2019.8761823

26. Z. Ding, et al, Application of non-orthogonal multiple access in LTE and 5G networks. IEEE Commun. Mag. 55(2), 185-191 (2017)

27. L. Dai, B. Wang, Y. Yuan, S. Han, I. Chih-lin, Z. Wang, Non-orthogonal multiple access for 5G: Solutions, challenges, opportunities, and future research trends. IEEE Commun. Mag. 53(9), 74-81 (2015)

28. W. Wu, X. Yin, P. Deng, T. Guo, B. Wang, Transceiver design for downlink SWIPT NOMA systems with cooperative full-duplex relaying. IEEE Access. 7, 33464-33472 (2019)

29. Q. Liu, T. LV, Z. Lin, Energy-efficient transmission design in cooperative relaying systems using NOMA. IEEE Commun. Lett. 22(3), 1-1 (2018)

30. M. F. Kader, S. Y. Shin, V. C. M. Leung, Full-duplex non-orthogonal multiple access in cooperative relay sharing for $5 \mathrm{G}$ systems. IEEE Trans. Veh. Technol. 67(7), 1-1 (2018)

31. J. Sun, Z. Wang, Q. Huang, Cyclical NOMA based UAV-enabled wireless network. IEEE Access. 7, 4248-4259 (2019)

32. M. F. Sohail, C. Y. Leow, S. H. Won, Non-orthogonal multiple access for unmanned aerial vehicle assisted communication. IEEE Access, 22716-22727 (2018). https://doi.org/10.1109/access.2018.2826650

33. T. Qi, W. Feng, Y. Wang, Outage performance of non-orthogonal multiple access based unmanned aerial vehicles satellite networks. China Commun. 15(5), 1-8 (2018)
34. C. Li, F. Sun, J. M. Cioffi, L. Yang, Energy efficient MIMO relay transmissions via joint power allocations. IEEE Trans. Circ. Syst. 61(7), 531-535 (2014)

35. W. Wu, F. Zhou, R. Q. Hu, B. Wang, Energy-efficient resource allocation for secure NOMA-enabled mobile edge computing networks. arXiv:1910.09886 (2019)

36. L. R. Varshney, Transporting information and energy simultaneously. IEEE Int. Symp. Inform. Theory, 1612-1616 (2008). https://doi.org/10.1109/isit. 2008.4595260

37. L. Liu, R. Zhang, K. C. Chua, Wireless information and power transfer: a dynamic power splitting approach. IEEE Trans. Commun. 61(9), 3990-4001 (2013)

38. K. Huang, E. Larsson, Simultaneous information and power transfer for broadband wireless systems. IEEE Trans. Sig. Process. 61(23), 5972-5986 (2013)

39. A. A. Nasir, X. Zhou, S. Durrani, R. A. Kennedy, Relaying protocols for wireless energy harvesting and information processing. IEEE Trans. Wirel. Commun. 12(7), 3622-3636 (2013)

40. W. Wu, B. Wang, Robust secrecy beamforming for wireless information and power transfer in multiuser MISO communication system. EURASIP J. Wirel. Commun. Netw. 1(2015), 161 (2015)

41. S. Yin, Y. Zhao, L. Li, F. R. Yu, UAV-assisted cooperative communications with power-splitting Information and power transfer. IEEE Trans. Green Commun. Netw. 3(4), 2400-2473 (2019)

42. D. N. K. Jayakody, T. D. P. Perera, M. C. Nathan, M. Hasna, in 2019 IEEE International Black Sea Conference on Communications and Networking (BlackSeaCom). Self-energized full-duplex UAV-assisted cooperative communication systems, (2019), pp. 1-6. https://doi.org/10.1109/ blackseacom.2019.8812864

43. J. Xu, Y. Zeng, R. Zhang, in 15th International Symposium on Wireless Communication Systems (ISWCS). UAV-enabled wireless power transfer with directional antenna: a two-user case (invited paper), (2018), pp. 1-6. https://doi.org/10.1109/iswcs.2018.8491102

44. C. Li, H. J. Yang, F. Sun, J. M. Cioffi, L. Yang, Adaptive overhearing in two-way multi-antenna relay channels. IEEE Sig. Process. Lett. 23(1), $117-120(2016)$

45. M. Kim, J. Lee, Outage probability of UAV communications in the presence of interference (2018). https://doi.org/10.1109/glocom.2018.8647521

46. C. Li, P. Liu, C. Zou, F. Sun, J. M. Cioffi, L. Yang, Spectral-efficient cellular communications with coexistent one- and two-hop transmissions. IEEE Trans. Veh. Technol. 65(8), 6765-6772 (2016)

47. H. H. M. Tam, et al, Successive convex quadratic programming for quality-of-service management in full-duplex MU-MIMO multicell networks. IEEE Trans. Commun. 64(6), 2340-2353 (2016)

48. B. R. Marks, G. P. Wright, A general inner approximation algorithm for nonconvex mathematical programs. Oper. Res. 26(4), 681-683 (1978)

\section{Publisher's Note}

Springer Nature remains neutral with regard to jurisdictional claims in published maps and institutional affiliations.

\section{Submit your manuscript to a SpringerOpen ${ }^{\odot}$ journal and benefit from:}

- Convenient online submission

- Rigorous peer review

- Open access: articles freely available online

- High visibility within the field

- Retaining the copyright to your article

Submit your next manuscript at $>$ springeropen.com 